\title{
Use of autologous fibrin glue for endoscopic treatment of esophageal lesions
}

Authors

Institution
Matthias Lucas, Petra Seeber

The Institute for Blood Management, Gotha, Germany
Bibliography

DOI http://dx.doi.org/

$10.1055 / \mathrm{s}-0034-1392107$

Published online: 24.6 .2015

Endosc Int Open 2015; 03:

E405-E408

(c) Georg Thieme Verlag KG

Stuttgart $\cdot$ New York

E-ISSN 2196-9736

Corresponding author

Matthias Lucas

The Institute for Blood

Management

99867Gotha

Germany

Fax: +49-3621-860802

Matthias_lucas@web.de
Background and study aims: Esophageal leaks are a potentially life-threatening condition. One treatment option is injection therapy with commercially available fibrin glue. We describe herein

\section{Introduction}

\section{$\nabla$}

Esophageal leakage is a rare but potentially lifethreatening condition that requires interdisciplinary management. Potential treatment modalities consist of surgical revision with or without interponation of stomach or bowel, the use of covered stents, endoscopic clipping, vacuum therapy or the use of commercial fibrin glue with or without resorbable mesh implantation. At all times, drainage of a developing pleural and/ or mediastinal abscess as well as broad-spectrum antibiotic coverage and parenteral nutrition are mandatory. In instances in which endoscopic injection is chosen to seal esophageal leaks, fibrin glue seems to be the agent used most often. Autologous fibrin glue (or autologous platelet-rich plasma sealant) has been used for decades to improve wound healing and to reduce surgical blood loss $[1,2]$. In rare cases, it also has been used to close vesicovaginal fistulas [3]. To our knowledge, this is the first report about use of autologous fibrin glue to treat esophageal leaks by endoscopic injection.

\section{Case Reports \\ $\nabla$}

Patient 1

A patient developed an anastomotic leak approximately $1 \mathrm{~cm}$ in diameter with free perforation into the right pleural cavity after undergoing esophageal resection due to cardia carcinoma ( $\bullet$ Fig.1-3). Discussing the treatment options with the patient, a surgical intervention was thought to be too dangerous because the patient was severely anemic; as a Jehovah's Witness, did not authorize the use of blood products; [4] and a method to close esophageal leaks by injecting autologous fibrin glue prepared exclusively with the patient's own blood.

had already undergone surgical revision with interposition of colon for a prior ischemic loss of a small bowel interponate. Implanting a covered stent to close the lesion was deemed impossible since the conformation of the anastomotic site did not allow for safe placement of a stent and carried a high risk of stent dislodgement.

The patient consented to lavage and drainage of the chest cavity. That was performed immediately and the patient was placed on broad-spectrum antibiotics. Endoscopic treatment of the esophageal leak was then attempted, initially by clipping the lesion, but it proved technically challenging and did not succeed in closing the leak. Therefore, as a last resort, autologous fibrin glue ( $\bullet$ Fig.4) was prepared after the patient gave informed consent. The glue was prepared and $3 \times 1.5 \mathrm{~mL}$ of it was endoscopically injected submucosally around the leak ( $\bullet$ Fig. 5 and $\bullet$ Fig. 6 ) in three different places, as described in detail in $\square$ Table 1 . At each endoscopic session, the procedure was repeated. Injection sessions were scheduled for Monday, Wednesday, and Friday of 2 consecutive weeks. There was no clinical evidence of tissue inflammation or necrosis after injection. On the contrary, the wound edges seemed to regain vitality. Between injection sessions, the patient was allowed to drink water and tea with sugar or honey, which helped to clean the chest cavity and kept bacterial accumulation in the chest to a minimum. After the sixth injection session, the leak was closed, as demonstrated by cessation of output from the chest drain and radiologic studies with liquid contrast medium and methylene blue ( Fig.7 and $\bullet$ Fig.8). 


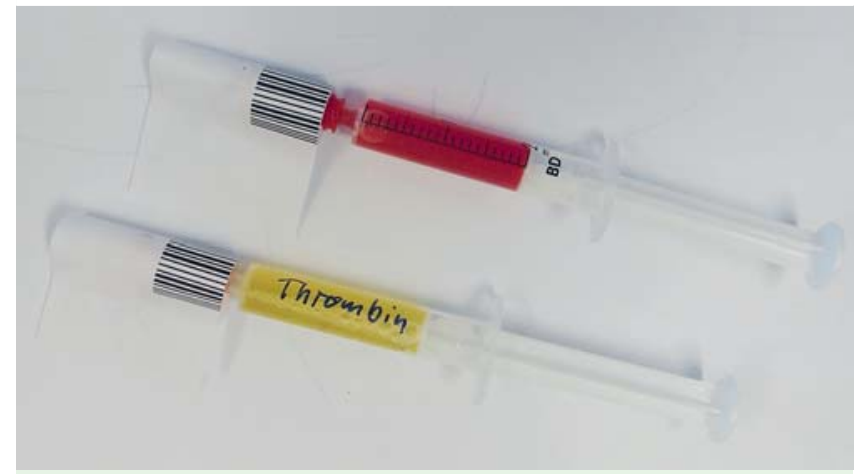

Fig. 1 Glue ready for use.

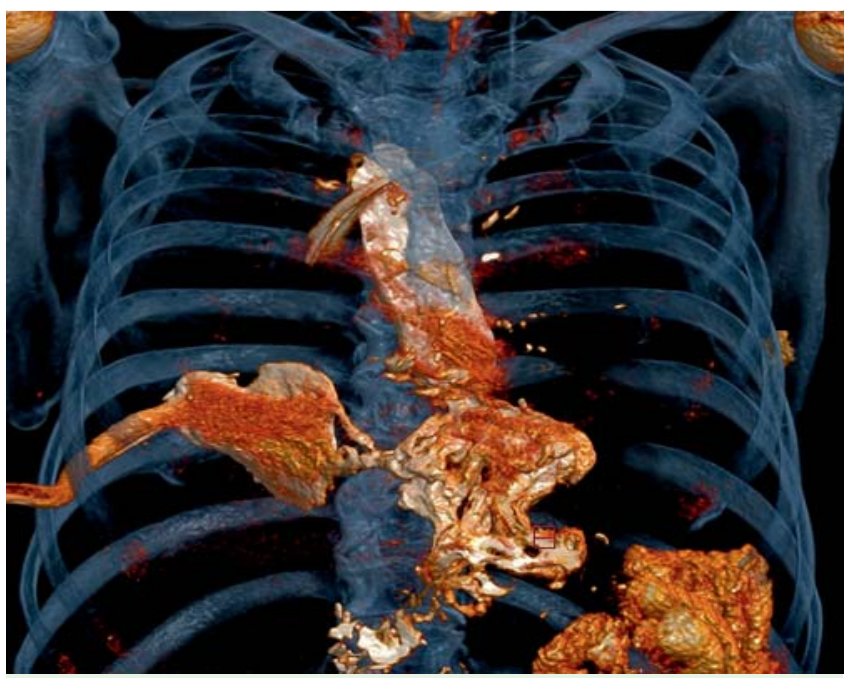

Fig. 3 3D-reconstruction of CAT-Scan.

\section{Patient 2}

A patient who suffered from gastric outlet stenosis had experienced an episode of violent vomiting, whereupon he was left dyspneic with severe pain in his right shoulder. During the following hours, he became septic and was admitted to the intensive care unit, where he was diagnosed with an esophageal tear of about $1.5 \mathrm{~cm}$ (Boerhave syndrome) with free perforation into the mediastinum. Neither the patient nor his attorney would consent to a surgical intervention on the esophagus, but they did permit chest and mediastinal lavage and drainage as well as endoscopic treatment. The patient also refused placement of a covered esophageal stent and the use of any blood product, including commercially available fibrin glue. We again decided to try endoscopic clipping and autologous fibrin glue injection. It took 11 injection sessions, each 2 days apart, to close the leak. Clipping did not prove to add to the success because the clips either got lost or failed to attach the wound edges in the fragile tissue. As in the first case presented here, no tissue inflammation or necrosis developed after injection and the wound edges regained vitality. After leak closure, the patient's recovery was uneventful and he was discharged to rehabilitation with a gastrojejunal tube (Trilumina ${ }^{\circledR}$ ) bridging the gastric outlet stenosis, which he did

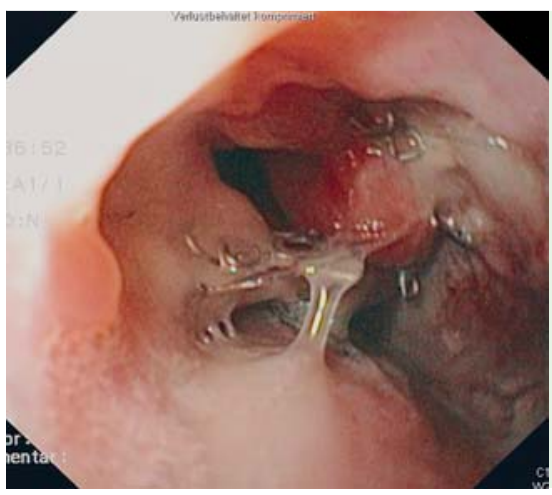

Fig.4 Endoscopic diagnosis of anastomotic lesion

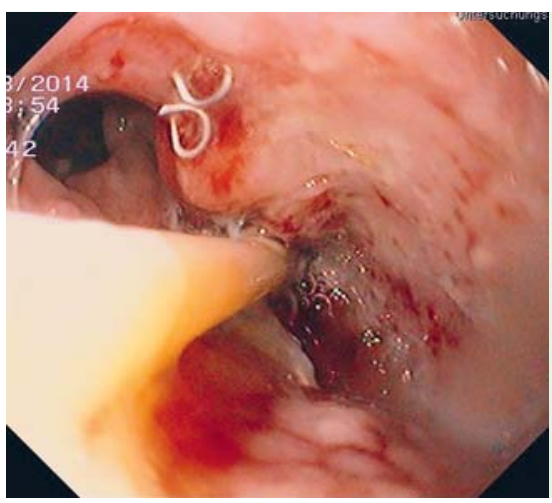

Fig. 5 Injection of autologous glue.

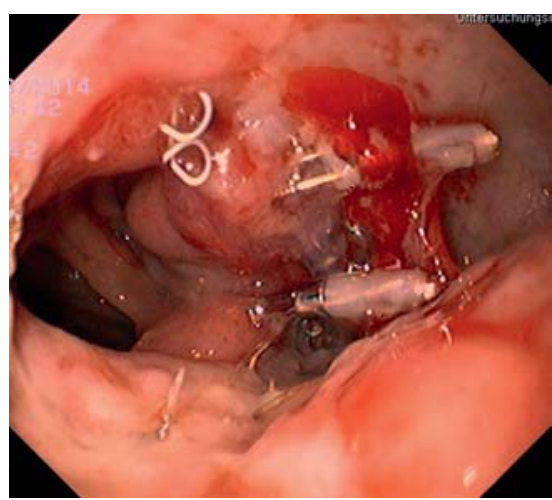

Fig. 6 After glue injection with residual glue on surface. 


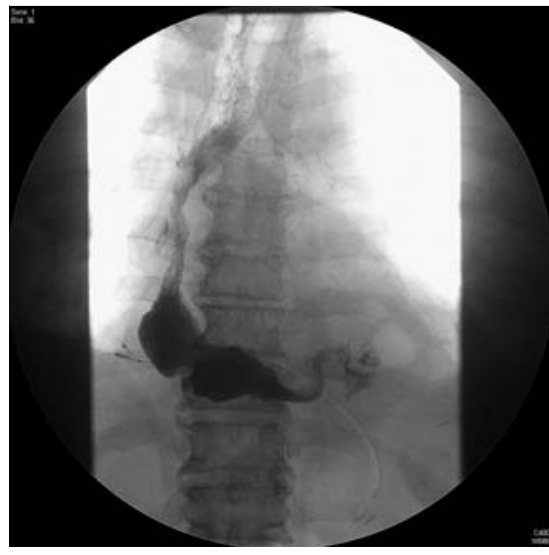

Fig. 7 Control x-ray after closure of leak.

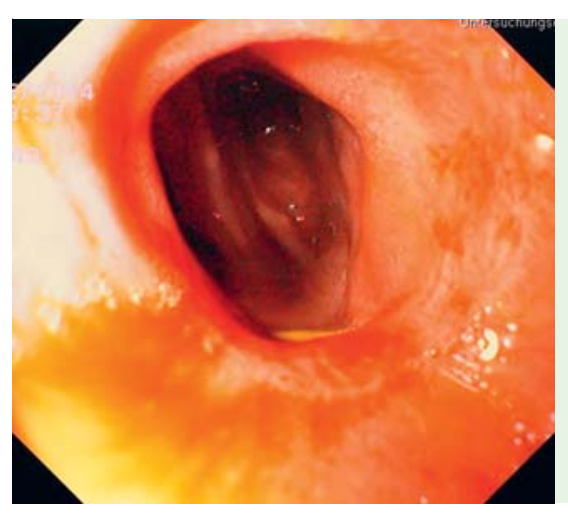

Fig. 8 Endoscopic picture two months after closure.

\section{Discussion}

\section{$\nabla$}

When surgical maneuvers, endoscopic placement of clips or stents or the placement of an endoscopic vacuum device are impossible because of medical conditions or are not permitted by a patient, medical personnel are left with endoscopic injection as the only therapeutic option for treatment of esophageal leakage [5]. However, if a patient does not consent to use of commercially available fibrin glue, treatment is a daunting task for the health care provider. Using glue made with something other than donated blood, rather than commercial fibrin glue, seems to be an alternative.

Many commercially available injectable sealants contain derivatives of donated blood, such as thrombin, and may not be acceptable to some patients. Other glues, such as cyanoacrylates, are not readily biodegradable and may impair tissue healing, with resultant reduced scar strength, tissue necrosis and recurrent anastomotic leakage [6]. Use of cyanoacrylate for closure of an esophageal leak has been reported only rarely and exclusively for small lesions of about 3 to $4 \mathrm{~mm}[7,8]$. Therefore, we were reluctant to inject cyanoacrylate submucosally around an esophageal leak. The most promising glue available in the above described situations seemed to be physiologically degradable autologous fibrin glue.

Apart from its coagulation potential, autologous glue contains autologous leukocytes and platelets and the contents of their granulae, which not only promote wound healing and revascularization but also have antibacterial properties $[9,10]$ that may be beneficial in treatment of a wound that is in contact with bacterially contaminated saliva.

Another advantage of autologous glue is the simplicity of preparation and its low costs. Glue preparation takes about 20 minutes
Table 1 Preparing a two-component autologous fibrin glue.

Collect the following standard laboratory materials:

- 3 sterile laboratory tubes with cap, fitted into a laboratory centrifuge (10-mL volume)

- 6 2-mL syringes

- 6 sterile caps for the syringes

- $310-\mathrm{mL}$ syringes

- 3 long cannulae

- 3 standard citrate blood tubes (2-mL green-top Monovettes ${ }^{\circledR}$

by Saarstedt) for a needed total volume of $6 \mathrm{~mL}$ whole blood in citrate

- 2 pairs of sterile gloves

- 1 sterile drape

- 1 vial of calcium gluconate $10 \%$

- 13 labels with the patient's name

- 1 laboratory centrifuge with adjustable rotation speed

\section{Preparation:}

- In a sterile fashion, draw $5 \mathrm{~mL}$ of the patient's own blood into a $10-\mathrm{mL}$ syringe and immediately transfer it into a sterile laboratory tube labeled "thrombin" and close the tube.

- Draw $6 \mathrm{~mL}$ of blood into the three $2-\mathrm{mL}$ citrate laboratory tubes.

- Shake the "thrombin" laboratory tube gently for 1 minute and allow the blood to clot (approximately 20 - 30 minutes).

- Meanwhile, spin the citrate blood for 8 minutes at a speed of $800 \mathrm{rpm}$ in the laboratory centrifuge, separating the blood into three components: red cells on the bottom, platelets on top of the red cell layer, and the plasma layer on top.

- After centrifugation, open the citrate tubes and transfer the platelet layer and the plasma into one of the sterile laboratory tubes labeled "PRP" (platelet-rich plasma) (using a long cannula and one of the 10 -mL syringes).

- Spin the tubes with "PRP" and "thrombin" again at a speed of $1200 \mathrm{rpm}$ for 8 minutes.

Label three 2-mL syringes with the patient's name and "PRP" and three

2-mL syringes with the patient's name and "thrombin".

To prepare the first component of the glue, draw three $1-\mathrm{mL}$ aliquots of the platelet-plasma-layer of the "PRP" tube into each of the $2-\mathrm{mL}$ syringes labeled "PRP" and cap the syringes.

To prepare the second component of the glue, draw three aliquots of $0.3-0.5 \mathrm{~mL}$ into each of the $2-\mathrm{mL}$ syringes labeled "thrombin." To that end, using a long cannula, squeeze as much liquid as possible out of the clot. Then, add $0.2 \mathrm{~mL}$ of the calcium solution to each "thrombin" syringe.

The glue components can be kept at room temperature for up to 6 hours before use ( Fig. 1).

Application:

- Use the shortest possible endoscope with the shortest possible needle. - Intubate the patient's esophagus, flush the leak with saline, and brush the wound edges with an endoscopic brush.

- Place the endoscopic needle about $1 \mathrm{~mm}$ submucosally next to the wound edge. In rapid sequence, sequentially inject one aliquot of the "PRP" component, one aliquot of the "thrombin," and saline in a volume equal to the dead-space volume of the endoscopic needle (use of a two-channel catheter may be preferable, if one is available).

- Redraw the needle and reinsert it at another site next to the wound edge and repeat the same procedure.

of laboratory time (not including the time to clot in the uncoagulated tube). The means to prepare the glue are found in nearly every hospital around the world that has a laboratory. The amount of blood needed for preparation is low compared to the use of most commercial machines for glue preparation $(11 \mathrm{~mL}$ vs. $50 \mathrm{~mL}$ ). That makes repeat preparation of glue feasible even when blood draws are restricted, as in patients for whom blood transfusions are not an option.

One drawback of autologous glue is its dependence upon a patient's own clotting capabilities. Because a patient's blood 
may either be hypercoagulable or hypocoagulable, clotting time and clot strength are unpredictable. In our experience, clotting time can range from a few seconds to 30 minutes, with an average of 2 minutes. Autologous glue also is rather liquid because it is not as concentrated as commercial fibrin glue. Thus applying the glue to a moving surface is difficult and clotting may be prolonged due to dilution with saliva or other surface fluids. Injection of the glue submucosally does circumvent these problems. With the injection technique reported here, which was also described for commercially available fibrin glue, autologous fibrin glue seems to be as effective as commercially available fibrin glues for sealing esophageal leaks. That is evidenced by comparison of the time from first injection to closure of a leak. In the medical literature, 1 to 40 injection sessions are reported to be necessary to close anastomotic leaks [8]. We needed 6 and 11 sessions of autologous glue injection, respectively, to close the leaks in our patients, which is well within the range described for commercial glue. It may, however, have taken fewer injection sessions if glue injection had been combined with a Vicryl plug.

\section{Conclusion}

$\nabla$

Endoscopic injection therapy with fibrin glue for esophageal leaks has proven a feasible alternative to surgical repair. While the use of commercially available fibrin glue is currently standard of care, the use of autologous glue seems to be a viable alternative in cases in which commercial fibrin glue is either unavailable or its use is undesirable to a patient.

Competing interests: The authors received fundings from Helios Research Center and Vifor for publishing and lecturing respectively.

\section{References}

1 Giuffrè G, Caputo G, Misso $S$ et al. Platelet-rich plasma treatment and hemostasis in patients with hemorrhagic risk. Minerva Stomatol 2006; 55: 599-609

2 Yilmaz S, Aksoy E, Doganci S et al. Autologous platelet-rich plasma in treatment of chronic venous leg ulcers: A prospective case series. Vascular 2014

3 Shirvan MK, Alamdari DH, Ghoreifi A. A novel method for iatrogenic vesicovaginal fistula treatment: autologous platelet rich plasma injection and platelet rich fibrin glue interposition. J Urol 2013; 189: $2125-$ 2129

4 www.jw.org/en/bible-teachings/questions/bible-about-blood-transfusion/

5 Victorzon M, Victorzon S, Peromaa-Haavisto P. Fibrin glue and stents in the treatment of gastrojejunal leaks after laparoscopic gastric bypass: A case series and review of the literature. Obes Surg 2013; 23: $1692-$ 1697

6 Singer AJ, Thode HCJr. A review of the literature on octylcyanoacrylate tissue adhesive. Am J Surg 2004; 187: 238-248

7 Pramateftakis MG, Vrakas G, Kanellos I et al. Endoscopic application of n-butyl-2-cyanoacrylate on esophagojejunal anastomotic leak: a case report. J Med Case Reports 2011; 5: 96

8 Kotzampassi K, Eleftheriadis E. Tissue sealants in endoscopic applications for anastomotic leakage during a 25-year period. Surgery 2015; 157: $79-86$

9 Yang $L C, H u$ SW, Yan $M$ et al. Antimicrobial Activity of Platelet-Rich Plasma and Other Plasma Preparations Against Periodontal Pathogens. J Periodontol 2014: 1-17

10 Hamman BL, Stout $L Y$, Theologes TT et al. Relation between topical application of platelet-rich plasma and vancomycin and severe deep sternal wound infections after a first median sternotomy. Am J Cardiol 2014; 113: 1415 - 1419 